\title{
Species-environment relationship in the herb-subshrub layer of a moist Savanna site, Federal District, Brazil
}

\author{
Munhoz, CBR. ${ }^{\mathrm{a} *}$, Felfili, JM. ${ }^{\mathrm{b}}$ and Rodrigues, $C .^{\mathrm{c}}$ \\ aCurso de Ciências Biológicas, Universidade Católica de Brasília - UCB, \\ QS 07 Lote 01, Bloco E, Sala 10, CEP 72030-170, Taguatinga Sul, DF, Brazil \\ ${ }^{b}$ Departamento de Engenharia Florestal, Universidade de Brasília - UnB, \\ CP 04357, CEP 70919-970, Brasília, DF, Brazil \\ 'Laboratório Solocria, Av. Goiás, n. 5106, Qd. B Lt 08, Setor Urias Guimarães, CEP 74565-250 Goiânia, GO, Brazil \\ *e-mail: cassia@ucb.br \\ Received February 9, 2006 - Accepted April 11, 2006 - Distributed February 29, 2008
}

(With 2 figures)

\begin{abstract}
The soils are seasonally or permanently saturated in the moist grassland savanna, locally known as Campo Limpo Úmido. Soil moisture variation seems to determine spatial distribution of communities. The objective of this study is to analyse the relationship between environmental variables and the patterns of spatial distribution of species in the herbaceous-subshrub layer of an area of moist grassland at the Água Limpa Farm, Brasília, DF (15 $56^{\prime}$ to $15^{\circ} 59^{\prime} \mathrm{S}$ and $47^{\circ} 55^{\prime}$ to $47^{\circ} 58^{\prime} \mathrm{W}$ Gr.). An area of $400 \times 400 \mathrm{~m}$ was divided into four sections of $200 \times 200 \mathrm{~m}$ where four transects were randomly sampled. A line intercept method was adopted for the phytossociological study. Superficial soils samples $(0-20 \mathrm{~cm})$ were collected for chemical and textural analyses. Gravimetric soil moisture was measured quarterly during the study-year. A total of 85 species in 67 genera and 24 families were found. The diversity was high, Shannon's index, H', was 2.60 nats.cover ${ }^{-1}$. Floristic composition of the transects in soils with a high gravimetric soil moisture and high content of organic matter and sand differed from those transects in soils with a lower gravimetric soil moisture indicating seasonal variation. A Canonical Correspondence Analysis (CCA) showed significant correlations between soil texture and soil moisture features and species distribution. Gravimetric soil moisture, organic matter, clay, silt and sand were significantly correlated to species distribution in the moist grassland determining mosaics in the vegetation.
\end{abstract}

Keywords: moist grassland, herbaceous layer, phytosociology, line intercept method, cerrado.

\section{Variáveis ambientais e a distribuição de espécies do estrato herbáceo-subarbustivo em uma área de campo limpo úmido no Distrito Federal, Brasil}

\begin{abstract}
Resumo
O solo é sazonal ou permanentemente saturado nas savanas campestres úmidas, localmente conhecidas como Campo Limpo Úmido. A variação na umidade do solo parece determinar a distribuição espacial desta comunidade. O objetivo deste estudo foi analisar a relação entre as variáveis ambientais e o padrão de distribuição espacial das espécies do estrato herbáceo-subarbustivo em uma área de Campo Limpo Úmido na Fazenda Água Limpa, Brasília, DF (15²5' a $15^{\circ} 59^{\prime} \mathrm{S}$ e $47^{\circ} 55^{\prime}$ a $47^{\circ} 58^{\prime}$ 'WGr.). Uma área de 400 x $400 \mathrm{~m}$ foi subdividida em quatro porções de 200 x $200 \mathrm{~m}$ onde foram sorteadas as linhas de amostragem. No levantamento, fitossociológico adotou-se o método de inventário por interseção na linha. Foram coletadas amostras de solo superficial $(0-20 \mathrm{~cm})$ para análise química e textural. A umidade gravimétrica do solo foi obtida quinzenalmente durante o ano de estudo. Foram amostradas 85 espécies incluídas em 67 gêneros e 24 famílias. A diversidade da área segundo o índice de Shannon foi de H' = 2,60 nats.cobertura ${ }^{-1}$. A composição florística dos transectos em solos com alta umidade gravimétrica, altos teores de matéria orgânica e areia diferiu da dos transectos em solos com menor umidade gravimétrica e com variação sazonal na umidade. Uma análise de correspondência canônica (CCA) mostrou correlações significativas entre a textura e a umidade do solo e a distribuição das espécies. As variáveis ambientais mais fortemente correlacionadas foram umidade gravimétrica, matéria orgânica, argila, silte e areia, que foram preponderantes na determinação da distribuição das espécies do campo limpo úmido da FAL, determinando a formação de mosaicos na vegetação.
\end{abstract}

Palavras-chave: campo limpo úmido, estrato herbáceo, fitossociologia, método de interseção na linha, cerrado. 


\section{Introduction}

The Cerrado Biome originally occupied $23 \%$ of Brazil's land area (Ratter et al., 1997) covering the Brazilian Central Pateaux and presenting a wide physiognomic range, from grassland to tall woodlands where most physiognomies fit the definition of tropical savanna (Sarmiento, 1983).

Campos limpos are savanna grassland vegetation with less than $10 \%$ tree cover (Ribeiro and Walter, 1998). Their soils show a soil moisture gradient, some patches are swampy where the vegetation is named campo limpo úmido or moist grassland (Sarmiento, 1983), especially around springs and on the bottom-valleys while others are better-drained with shallow soils inhibiting tree growth (Eiten, 1972; Ribeiro and Walter, 1998). According to Reatto et al. (1998) $2.3 \%$ of the c. 2 million ha of the Cerrado is occupied by moist grasslands on hydromorphic soils plus $0.2 \%$ of this formation growing on humic gleys soils.

The herbaceous subshrub layer is an important component in all physiognomies of the Cerrado vegetation, from Grasslands (Campo Limpo) to Woodlands (Cerrado sensu stricto and Cerradão) (see Eiten, 1972 for a description of those physiognomies). It is the layer richest in species (Felfili et al., 1994; Mendonça et al., 1998), with more than 90 million ha of native vegetation used for pasture (Haridasan, 1996). Even so, little however is known about the nutrient contents, growth patterns and the natural distribution of the species, floristics, structure and dynamics of this ecosystem as well as the effect of disturbances such as fire, logging, clear-cutting, herbivory and extractivism, to guide conservation and management policies (Guimarães et al., 2002; Finlayson and van der Valk, 1995; Naranjo, 1995).

Knowledge of the species distribution patterns at a site can contribute to the understanding of the main environmental determinants of the community structure and functioning (Felfili, 1998). Some studies on the herbaceous subshrub layer of the cerrado (savanna woodland) vegetation suggest marked changes in species composition from one site to another (Mantovani and Martins, 1993; Felfili et al., 1998).

The only study based on multivariate techniques on grassland communities in Central Brazil was conducted by Goldsmith (1974) when he studied several sites covered by grassland vegetation in Mato Grosso concluding that they could be classified in two major types: Dry grasslands, those that occur over shallow soils on sandstone hills and lateritic outcrops within areas dominated by savanna woodland vegetation (cerrado) and moist grasslands that occur in valleys generally around gallery forests. He concluded that soil moisture seemed to be the main determinant of the vegetational variation with almost every species having a different range of soil moisture tolerance with some presenting a very narrow while others a very wide range.

In this work we assume that there is large spatial variation of species within the moist grassland related to a soil moisture gradient. The objective was to analyse the relationship between environmental variables and species distribution in an herbaceous subshrub layer of moist grassland in the Água Limpa Farm, Brasília, DF, Brazil.

\section{Material and Methods}

\subsection{Study site}

This study was conducted in a moist grassland at the Água Limpa Farm - FAL ( $15^{\circ} 56^{\prime}$ to $15^{\circ} 59^{\prime} \mathrm{S}$ and $47^{\circ} 55^{\prime}$ to $47^{\circ} 58^{\prime} \mathrm{W}$ Gr.), the University of Brasília Experimental Station. The study-area $\left(15^{\circ} 55^{\prime} 35.4\right.$ " to $15^{\circ} 56^{\prime} 4.1^{\prime \prime} \mathrm{S}$ and $47^{\circ} 54^{\prime} 20.8^{\prime \prime}$ to $47^{\circ} 54^{\prime} 21.9^{\prime \prime}$ WGr.) borders the Taquara stream gallery forest. This area suffered an accidental burn during the first week of August 1999, three months prior to the beginning of this study.

The soils are hydromorphic with the water table close to the surface, permanently saturated in the depressions (Humic Gley soils) and seasonally saturated in the remaining areas (Low Humic Gley soil), according to EMBRAPA (1999) soils classification.

The climate is Aw following Köppen with two well-defined seasons: one hot and rainy (from October to April) and the other colder and drier (from May to September). Average maximum temperature is $28.5{ }^{\circ} \mathrm{C}$ and the average minimum $12.0^{\circ} \mathrm{C}$. Average annual precipitation during the studied period (from November 1999 to December 2000) was $1500 \mathrm{~mm}$, according to the IBGE Climatological Station beside the FAL.

\subsection{Vegetation survey}

An area of $400 \times 400 \mathrm{~m}$ of moist grassland was divided in four sections of $200 \times 200 \mathrm{~m}$. At each section one transect, named $\mathrm{Ca} 0, \mathrm{Ca} 1, \mathrm{Ca} 2$ and $\mathrm{Ca} 3$, was randomly located in the direction perpendicular to the border of the Taquara stream gallery forest. Each transect was subdivided in sections of $1 \mathrm{~m}$. The size of the transects was based on a species-area curve (Müeller-Dombois and Ellenberg, 1974), when the curve leveled off, the sampling of transect ended. In the first section, the curve leveled off at $40 \mathrm{~m}(\mathrm{Ca} 0)$, the second section at $25 \mathrm{~m}(\mathrm{Ca} 1)$, the third at $30 \mathrm{~m}(\mathrm{Ca} 2)$ and the fourth at $25 \mathrm{~m}$ (Ca3). The leveling off of the species-area curve indicates that the transects offered a good floristic representation of the study-area.

The first phytosociological survey was conducted in November 1999, and the following sampling periods were in April, July, October and December 2000.

The line intercept method (Canfield, 1941, 1950) was used to sample the floristic composition and the linear coverage of the species. This method consists of projecting transects over the vegetation and recording the projection of each species over it. The length of the transect covered by a species divided by the length covered by all species is used to estimate the proportion of the area covered by that species, named cover. The frequency of 
species interception by segment was also recorded and named frequency.

In this study each transect was located in the field and subdivided in sections of $1 \mathrm{~m}$ with iron sticks. Those $1 \mathrm{~m}$-section were considered the sampling units (SU) for the phytosociological analyses. A wooden stick graduated in mm was placed over each SU for the evaluation of the horizontal projection of herbaceous subshrubs plants over the transect. The horizontal projection of each species over each SU along the sampling line was registered and absolute and relative cover were calculated as follow:

Absolute Cover $=$ sum of the horizontal projection of each species over all UAs sampled in the study-area.

Relative Cover $=$ absolute Cover of a species divided by the sum of the absolute cover of all species sampled in the study-area multiplied by 100 .

The frequency and the projection of each species in each segment was recorded for each transect, totaling 120 SU sampled. The frequency of species by segment was used in the matrix for Canonical analysis.

\subsection{Floristic diversity}

Shannon's (H') index was used to evaluate alpha diversity with the cover values from the five sampling occasions were used as variables. Shannon's index considers the proportional abundance of species in a sample using the proportion of individuals per species for its calculation (Margurran, 1988). Considering that abundance can be also evaluated by other measures such as frequency, basal area and cover (Kent and Coker, 1992) as well as for the number of individual we opted to use cover as a variable. Therefore, the proportion of cover of a species was used to calculate Shannon's index for grassland. That was done so because the definition of an individual is difficult with radicular expansions of the same individual (ramets) being common and difficult to distinguish above ground.

The species were identified with the use of specialized literature, by taxonomists and by comparison with vouchers deposited at the herbaria of the Universidade de Brasília - UB and the Reserva Ecológica do Instituto Brasileiro de Geografia e Estatística - IBGE. Vouchers collected were deposited in both herbaria.

\subsection{Soil sampling}

Superficial $500 \mathrm{~g}$-soil samples $(0-20 \mathrm{~cm})$ were collected every $5 \mathrm{~m}$ alongside the vegetation sampling transect. Chemical ( $\mathrm{pH}$; $\mathrm{Ca}+\mathrm{Mg}, \mathrm{Ca}, \mathrm{Mg}, \mathrm{Al}, \mathrm{H}+\mathrm{Al}, \mathrm{K}$, P, S, Na, Co, Zn, B, Cu, Fe, Mn and Mo contents; saturation of bases; Al saturation; organic matter; Cations Exchange Capacity (CTC)) and textural (clay, silt and sand contents) analyses were conducted according to EMBRAPA's (1997) protocol. The results of each 5-m soil samples were attributed to the respective set of five 1-m vegetation sampling units to build the environment matrix for Canonical analyses.

Fresh $(0-20 \mathrm{~cm})$ soil samples were taken at quarterly intervals during the study-period to obtain gravimet- ric soil moisture. The samples were collected at every 1-m section from transects $2 \mathrm{~m}$ distant and parallel to each of the transects where the vegetation was sampled to avoid damage to the area where the vegetation was monitored. The soil samples were put in sealed tins and stored in polystyrene boxes to be taken to the University of Brasília Forest Management Laboratory for weighting with scales at $0.01 \mathrm{~g}$ of precision, dried at $110{ }^{\circ} \mathrm{C}$ for 24 hours and weighted again to obtain the gravimetric soil moisture (EMBRAPA, 1997). Mean annual gravimetric soil moisture by $1-\mathrm{m}$ vegetation sampling units were used in the environment matrix for the Canonical Analyses.

\subsection{Correlation species $\mathrm{x}$ environmental variables}

A Canonical Correspondence Analysis (CCA) using the program CANOCO for Windows version 4 (ter Braak and Smilauer, 1998) was used to analyze the relationship between species distribution and environmental variables. A logarithmic transformation (ter Braak and Smilauer, 1998) was applied to the data. Graphs were drawn using CANODRAW 3.0 (Smilauer, 1992). The species matrix included only the 48 species with relative frequency equal or superior to ten. The environmental variables matrix included 21 soil variables. However, $\mathrm{Ca}+\mathrm{Mg}, \mathrm{Mg}, \mathrm{P}, \mathrm{H}+\mathrm{Al}$, CTC, bases saturation (V) and $\mathrm{Al}$ saturation presented a high redundancy (Variance Inflation Factor $>20$ ). Therefore, only the variables $\mathrm{Ca}, \mathrm{K}, \mathrm{pH}, \mathrm{Al}, \mathrm{Na}, \mathrm{Zn}, \mathrm{S}, \mathrm{Mn}, \mathrm{Fe}$, organic matter, clay, silt, sand and gravimetric soil moisture remained in the final analysis. A Monte Carlo (ter Braak and Smilauer, 1998) significance test was used to evaluate if both ordination axes were related to the environmental variables.

\section{Results}

\subsection{Soils}

The values of $\mathrm{pH}$ varied little between the transects (3.6 to 4.0) indicating a very acidic soil. Aluminum was high, varying from 0.4 to $1.4 \mathrm{cmolc} . \mathrm{dm}^{-3}$ between the transects. Calcium and Magnesium contents were low ( 0.3 to 0.4 cmolc. $\mathrm{dm}^{-3}$ ), with little variation within the same transect, except for $\mathrm{Ca}$ 0. Phosphorus was low (2.0

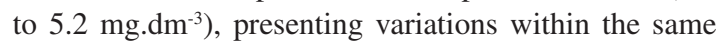
transect and between transects. Large differences were found for $\mathrm{K}$ within $\mathrm{Ca} 0$ but the contents overall were low $\left(0.06\right.$ a $\left.1.0 \mathrm{mg} \cdot \mathrm{dm}^{-3}\right)$. Base saturation was very low and Aluminum saturation varied from high to very high. The range was narrow for the soil variables within the same transect suggesting a homogeneity at that scale (Table 1). $\mathrm{Ca} 0$ soil was loamy-sand while $\mathrm{Ca} 3$ soils were sandy loam and the soils of the other two transects were loamy clay sand (Table 1).

Gravimetric soil moisture was high with the values of transects $\mathrm{Ca} 0$ and $\mathrm{Ca} 3$ higher than those of $\mathrm{Ca} 1$ and $\mathrm{Ca} 2$ (Table 1). The average of all transects varied little during the studied period. 
Table 1. Chemical and granulometric characteristics of 16 soil samples $(0-20 \mathrm{~cm})$ in a moist grassland (Seasonally saturated tropical grassland) site of the Água Limpa Farm, Brasília, DF. Average values aregiven and amplitudes in brackets of the $n$ samples in each sampling transect. G.S.M. = Gravimetric soil moisture.

\begin{tabular}{|c|c|c|c|c|c|}
\hline Transect & n & $\mathrm{Ca}\left(\mathrm{cmolc} . \mathrm{dm}^{-3}\right)$ & $\operatorname{Mg}\left(\mathrm{cmolc} \mathrm{dm}^{-3}\right)$ & $\mathrm{Fe}\left(\mathrm{mg} \mathrm{dm}^{-3}\right)$ & H+Al $\left(\right.$ molc.dm $\left.{ }^{-3}\right)$ \\
\hline $\mathrm{Ca} 0$ & 5 & $0.3(0.3)$ & $0.1(0.1)$ & 180.3(196.0) & $9.2(3.7)$ \\
\hline Ca1 & 3 & $0.2(0)$ & $0.1(0)$ & $30.6(14.0)$ & $14.8(3.1)$ \\
\hline $\mathrm{Ca} 2$ & 5 & $0.2(0)$ & $0.1(0)$ & $59.6(32.8)$ & $8.9(4.9)$ \\
\hline \multirow[t]{2}{*}{$\mathrm{Ca} 3$} & 3 & $0.2(0)$ & $0.1(0)$ & $22.4(13.8)$ & $14.5(3.4)$ \\
\hline & & K (cmolc.dm $\left.{ }^{-3}\right)$ & $S\left(\mathrm{mg.dm}^{-3}\right)$ & Al saturation $(\%)$ & $\mathrm{Na}\left(\mathrm{mg} \mathrm{dm}^{-3}\right)$ \\
\hline $\mathrm{Ca} 0$ & 5 & $0.07(0.09)$ & $2.1(0.8)$ & $60.6(19.3)$ & $2.8(1.0)$ \\
\hline Ca1 & 3 & $0.1(0.06)$ & $2.4(0.7)$ & $77.0(4.2)$ & $2.3(1.0)$ \\
\hline $\mathrm{Ca} 2$ & 5 & $0.09(0.9)$ & $2.5(1.5)$ & $47.6(9.1)$ & $2.0(0.0)$ \\
\hline \multirow[t]{2}{*}{$\mathrm{Ca} 3$} & 3 & $0.06(0.04)$ & $2.2(0.6)$ & $73.9(3.9)$ & $2.3(0.1)$ \\
\hline & & Co $\left(\mathrm{mg} \mathrm{dm}^{-3}\right)$ & Zn (mg.dm-3 $)$ & B $\left(\mathbf{m g . d m}^{-3}\right)$ & $\operatorname{Mo}\left(\mathbf{m g . d m}^{-3}\right)$ \\
\hline $\mathrm{Ca} 0$ & 5 & $0.07(0.05)$ & 7.1(3.0) & $0.2(0.1)$ & $0.08(0.03)$ \\
\hline Ca1 & 3 & $0.06(0.03)$ & $5.6(3.3)$ & $0.1(0.04)$ & $0.07(0.02)$ \\
\hline $\mathrm{Ca} 2$ & 5 & $0.07(0.02)$ & $3.1(6.8)$ & $0.2(0.1)$ & $0.08(0.02)$ \\
\hline \multirow[t]{2}{*}{$\mathrm{Ca} 3$} & 3 & $0.07(0.04)$ & $4.5(1.1)$ & $0.1(0.04)$ & $0.08(0.02)$ \\
\hline & & Mn $\left(\right.$ mg.dm $\left.^{-3}\right)$ & $\mathrm{Cu}\left(\mathrm{mg.dm}^{-3}\right)$ & $\mathrm{pH}\left(\mathrm{CaCl}_{2}\right)$ & Al $\left(\mathrm{cmolc} \mathrm{dm}^{-3}\right)$ \\
\hline $\mathrm{Ca} 0$ & 5 & $1.2(1.5)$ & $0.4(0.4)$ & $3.7(0.2)$ & $0.7(0.2)$ \\
\hline Ca1 & 3 & $3.8(4.0)$ & $0.4(0.3)$ & $3.7(0.1)$ & $1.4(0.2)$ \\
\hline $\mathrm{Ca} 2$ & 5 & $1.6(2.1)$ & $0.6(0.6)$ & $4.0(0.2)$ & $0.4(0.1)$ \\
\hline \multirow[t]{2}{*}{$\mathrm{Ca} 3$} & 3 & $0.6(0.5)$ & $0.4(0.1)$ & $3.6(0.1)$ & $1.0(0.1)$ \\
\hline & & P - Mehlich (mg.dm $\left.{ }^{-3}\right)$ & CTC & Organic matter $(\%)$ & Base saturation (\%) \\
\hline $\mathrm{Ca} 0$ & 5 & $5.2(5.1)$ & $9.7(4.1)$ & $9.3(2.3)$ & $5.2(3.4)$ \\
\hline $\mathrm{Ca} 1$ & 3 & $3.0(2.9)$ & $15.2(3.1)$ & $8.8(1.4)$ & $2.7(0.4)$ \\
\hline $\mathrm{Ca} 2$ & 5 & $2.0(2.2)$ & $9.4(4.9)$ & $11.4(13.5)$ & $4.4(2.0)$ \\
\hline \multirow[t]{2}{*}{$\mathrm{Ca} 3$} & 3 & $3.1(3.8)$ & $15.1(3.4)$ & $16.0(9.0)$ & $2.5(0.9)$ \\
\hline & & G.S.M. (\%) & Clay $(\%)$ & Silt (\%) & Sand $(\%)$ \\
\hline $\mathrm{Ca} 0$ & 5 & $637.5(347.0)$ & $15.2(6.0)$ & $5.0(0.0)$ & $79.8(6.0)$ \\
\hline $\mathrm{Ca} 1$ & 3 & $90.4(80.2)$ & $33.3(4.0)$ & $9.0(2.0)$ & $57.7(4.0)$ \\
\hline $\mathrm{Ca} 2$ & 5 & $102.2(53.7)$ & $31.8(37.0)$ & $8.2(6.0)$ & $60.0(43.0)$ \\
\hline $\mathrm{Ca} 3$ & 3 & $492.1(267.3)$ & $10.3(2.0)$ & $4.6(1.0)$ & $85.0(3.0)$ \\
\hline
\end{tabular}

\subsection{Vegetation}

A total of 85 species in 67 genera and 24 families was found during the study-period (Table 2). Species richness varied between transects with 29 species found at $\mathrm{Ca} 0$ and 40, 45 and 27 at $\mathrm{Ca} 1, \mathrm{Ca} 2$ and $\mathrm{Ca} 3$, respectively. Only four species $(4.7 \%)$ were found at all transects (Table 2) while most species were restricted to just one transect (Table 2). Shannon's diversity index H' was 2.60 nats.cover ${ }^{-1}$ for the all community. Shannon's $\left(\mathrm{H}^{\prime}\right)$ were higher at $\mathrm{Ca} 1\left(\mathrm{H}^{\prime}=2.4\right)$ and $\mathrm{Ca} 2\left(\mathrm{H}^{\prime}=2.5\right)$ than in $\mathrm{Ca} 0\left(\mathrm{H}^{\prime} 1.8\right)$ and $\mathrm{Ca} 3\left(\mathrm{H}^{\prime}=1.7\right)$.

Grass (Poaceae) species showed the highest percent cover (CR) in the studied area, and Axonopus comans (Trin.) Henrard had the highest CR in all transects. This species represented more than $50 \%$ of the total cover at $\mathrm{Ca} 0$ and $\mathrm{Ca} 3$, transects with a higher gravimetric soil moisture throughout the year. Species with a high cover value varied along the transects (Table 2). Paspalum lineare Trin., Rhynchospora rugosa (Vahl) Galé and Arthropogon filifolius Filg. showed a high cover at $\mathrm{Ca} 0$ and Ca3 and Chromolaena vindex (DC.) R.M. King and H. Rob. and Mikania officinalis Mart. at $\mathrm{Ca} 1$ and $\mathrm{Ca} 2$. Some species restricted to one transect presented a high CR. The space not occupied by plants "empty soil" was high at $\mathrm{Ca} 0$ and $\mathrm{Ca} 2$ (Table 2).

Axonopus comans also presented a high relative frequency in all transects varying from $22.43 \%$ in $\mathrm{Ca} 3$ to $12.17 \%$ in $\mathrm{Ca} 2$, and being the second most frequent species $(13.75 \%)$ in $\mathrm{Ca} 1$ behind Chromolaena vindex with $14.39 \%$. Only 19 other species occurred with relative frequency higher than 5\%, they were: Lagenocarpus rigidus Nees, Syngonanthus gracilis (Bong.) Ruhland, Rhynchospora rugosa, Paspalum lineare, Paspalum polyphyllum Nees, Paspalum maculosum Trin, Ctenium brachystachyum (Nees) Kunth in Ca0; Hyptis carpinifolia Benth., Rhynchospora graminea Uittien and Andropogon lateralis Nees subsp. cryptopus (Trin. ex Hack.) A. 
Table 2. Relative cover (CR) and Relative frequency (FR) of the species in four sampling transects of the herbaceous-subshrub layer at a moist grassland site in the Água Limpa Farm. Brasília. DF. Bold = the highest CR at each sampling transect.

\begin{tabular}{|c|c|c|c|c|c|c|c|c|}
\hline \multirow[t]{2}{*}{ Species } & \multicolumn{2}{|c|}{ Ca0 } & \multicolumn{2}{|c|}{ Ca1 } & \multicolumn{2}{|c|}{$\mathrm{Ca} 2$} & \multicolumn{2}{|c|}{$\mathrm{Ca3}$} \\
\hline & $\mathbf{C R}$ & FR & CR & FR & CR & FR & $\mathbf{C R}$ & FR \\
\hline Axonopus comans (Trin.) Henrard & 54.47 & 20.04 & 25.26 & 13.75 & 23.11 & 12.17 & 58.48 & 22.43 \\
\hline Abolboda poarchon Seub. & - & - & - & - & - & - & 0.07 & 0.55 \\
\hline Achyrocline alata (Kunth) DC. & - & - & 0.03 & 0.13 & 0.27 & 0.96 & 0.06 & 0.37 \\
\hline Andropogon bicornis L. & - & - & 3.65 & 3.76 & 11.56 & 7.68 & - & - \\
\hline $\begin{array}{l}\text { Andropogon lateralis Nees subsp. cryptopus } \\
\text { (Trin. ex Hack.) A. Zanin. }\end{array}$ & 1.74 & 3.32 & 20.56 & 10.77 & 15.38 & 8.11 & 0.53 & 1.10 \\
\hline Andropogon leucostachyus Kunth & - & - & - & - & - & - & 1.11 & 2.76 \\
\hline Arthropogon filifolius Filg. & 2.11 & 2.47 & - & - & - & - & 7.49 & 8.64 \\
\hline Arthropogon villosus Nees & - & - & - & - & - & - & 6.58 & 7.72 \\
\hline Borreria latifolia (Aubl.) K. Schum. & - & - & 0.30 & 1.43 & - & - & - & - \\
\hline Borreria marticrovettiana E.L. Cabral & - & - & 0.03 & 0.26 & 0.05 & 0.21 & - & - \\
\hline Borreria tenella Cham. and Schltdl. & - & - & - & - & 0.31 & 1.71 & - & - \\
\hline Buchnera lavandulacea Cham. and Schldl. & - & - & 0.01 & 0.26 & - & - & - & - \\
\hline $\begin{array}{l}\text { Bulbostylis junciformis } \\
\text { Humb. Bonpl. and Kunth }\end{array}$ & - & - & - & - & 0.31 & 0.85 & - & - \\
\hline Bulbostylis sellowiana (Kunth) Palla & - & - & - & - & - & - & 0.13 & 0.37 \\
\hline Burmania flava Mart. & - & - & 0.01 & 0.13 & - & - & - & - \\
\hline Calea gardneriana Baker & - & - & - & - & - & - & 0.01 & 0.18 \\
\hline Cleistes sp. & 0.01 & 0.11 & - & - & - & - & - & - \\
\hline Clidemia capitellata (Bonpl.) D. Don & - & - & 0.69 & 1.17 & - & - & - & - \\
\hline Croton antisyphiliticus Mart. & - & - & 0.49 & 1.04 & 0.17 & 0.53 & - & - \\
\hline Ctenium brachystachyum (Nees) Kunth & 4.45 & 5.04 & - & - & - & - & - & - \\
\hline Ctenium cirrhosum (Nees) Kunth & - & - & - & - & - & - & 3.59 & 4.23 \\
\hline $\begin{array}{l}\text { Chamaesyce coecorum } \\
\text { (Mart. ex Boiss.) Croizat }\end{array}$ & - & - & - & - & 0.03 & 0.11 & - & - \\
\hline $\begin{array}{l}\text { Chromolaena vindex (DC.) } \\
\text { R.M.King and H.Rob. }\end{array}$ & - & - & 9.75 & 14.40 & 2.80 & 3.74 & - & - \\
\hline Deianira chiquitana Herzog & - & - & 0.02 & 0.26 & 0.06 & 0.53 & - & - \\
\hline Desmocelis villosa (Aubl.) Naudin & - & - & 0.12 & 0.65 & - & - & 0.16 & 1.29 \\
\hline Drosera montana A. St.-Hil. & 0.20 & 1.39 & - & - & 0.02 & 0.21 & - & - \\
\hline Echinolaena inflexa (Poir.) Chase & - & - & 0.09 & 0.78 & 7.45 & 5.66 & - & - \\
\hline Elionurus muticus (Spreng.) Kuntze & - & - & 1.73 & 1.04 & 2.08 & 1.71 & - & - \\
\hline bare soil & 1.52 & 2.04 & 1.43 & 2.59 & $\mathbf{5 . 5 0}$ & 7.36 & 0.54 & 0.92 \\
\hline Eryngium maginatum Pohl ex Urban & - & - & 0.15 & 0.52 & - & - & - & - \\
\hline Eriocaulaceae (CM-1817) (New species) & - & - & - & - & 0.24 & 1.17 & - & - \\
\hline Erythroxylum deciduum A. St.-Hil & - & - & 0.02 & 0.13 & - & - & - & - \\
\hline Hypogynium virgatum (Desv.) Dandy & - & - & 4.46 & 3.37 & 6.09 & 4.38 & 3.45 & 5.33 \\
\hline Hyptis carpinifolia Benth. & - & - & 13.68 & 11.15 & - & - & - & - \\
\hline Ipomoea procurrens Meisn. & - & - & - & - & 0.08 & 0.21 & - & - \\
\hline Lagenocarpus rigidus Nees & 9.83 & 12.22 & 0.21 & 0.52 & 0.37 & 0.53 & - & - \\
\hline Leandra polystachya (Naudin) Cogn. & - & - & 0.30 & 0.78 & - & - & - & - \\
\hline Lessingianthus grearii (H.Rob.) H.Rob. & - & - & - & - & 0.52 & 1.28 & - & - \\
\hline Mesosetum ferrugineum (Trin.) Chase & 0.01 & 0.11 & - & - & - & - & 1.29 & 2.57 \\
\hline Microlicia polystemma Naudin & - & - & 0.21 & 0.52 & - & - & - & - \\
\hline Mikania officinalis Mart. & - & - & 3.45 & 3.89 & 1.83 & 3.42 & - & - \\
\hline Monnina stenophylla A. St.-Hil. and Moq. & - & - & 3.11 & 3.11 & - & - & - & - \\
\hline
\end{tabular}


Table 2. Continued...

\begin{tabular}{|c|c|c|c|c|c|c|c|c|}
\hline \multirow[t]{2}{*}{ Species } & \multicolumn{2}{|c|}{ Ca0 } & \multicolumn{2}{|c|}{ Ca1 } & \multicolumn{2}{|c|}{$\mathrm{Ca} 2$} & \multicolumn{2}{|c|}{$\mathrm{Ca3}$} \\
\hline & $\mathbf{C R}$ & FR & $\mathbf{C R}$ & FR & $\mathbf{C R}$ & FR & $\mathbf{C R}$ & FR \\
\hline $\begin{array}{l}\text { Otachyrium seminudum } \\
\text { Hack. ex Send. and Soderstr. }\end{array}$ & - & - & - & - & 0.06 & 0.11 & - & - \\
\hline Panicum parvifolium Lam. & 0.20 & 0.97 & 0.67 & 2.34 & - & - & 0.09 & 0.74 \\
\hline Paspalum ellipticum Döll & - & - & - & - & - & - & 1.29 & 2.57 \\
\hline Paspalum imbricatum Filg. & - & - & 0.94 & 1.04 & - & - & - & - \\
\hline Paspalum lineare Trin. & 9.28 & 5.79 & 1.14 & 1.17 & 0.03 & 0.11 & 2.07 & 2.21 \\
\hline Paspalum maculosum Trin. & 2.61 & 5.25 & 0.69 & 0.65 & 1.06 & 0.64 & - & - \\
\hline Paspalum polyphyllum Nees & 3.52 & 5.36 & - & - & 9.00 & 6.83 & 0.28 & 1.29 \\
\hline Pfaffia jubata Mart. & - & - & 0.66 & 2.72 & 2.65 & 5.02 & - & - \\
\hline Piriqueta sidifolia Urb. & - & - & - & - & 0.02 & 0.21 & - & - \\
\hline Polygala carphoides Chodat & - & - & - & - & 0.06 & 0.43 & - & - \\
\hline Polygala gracilis Kunth. & - & - & - & - & 0.01 & 0.11 & - & - \\
\hline $\begin{array}{l}\text { Polygala hygrophyla } \\
\text { Humb. Bonpl. and Kunth }\end{array}$ & - & - & 0.03 & 0.13 & - & - & - & - \\
\hline $\begin{array}{l}\text { Polygala longicaulis } \\
\text { Humb. Bonpl. and Kunth }\end{array}$ & - & - & 0.03 & 0.65 & 0.45 & 3.84 & - & - \\
\hline Polygala tenuis DC. & - & - & - & - & 0.01 & 0.11 & - & - \\
\hline Rhynchanthera grandiflora (Aubl.) DC. & - & - & 0.07 & 0.55 & 0.07 & 0.55 & 0.07 & 0.55 \\
\hline $\begin{array}{l}\text { Rhynchospora cf. albiceps } \\
\text { Humb. Bonpl. and Kunth }\end{array}$ & 0.52 & 1.18 & - & - & 0.71 & 2.24 & 0.07 & 0.18 \\
\hline Rhynchospora emaciata (Nees) Böeck. & 0.58 & 1.72 & - & - & 1.56 & 2.78 & 0.41 & 1.47 \\
\hline $\begin{array}{l}\text { Rhynchospora globosa (Humb. Bonpl. and } \\
\text { Kunth) Roem. and Schult. }\end{array}$ & 0.72 & 2.57 & - & - & 0.80 & 2.78 & 2.81 & 2.76 \\
\hline Rhynchospora graminea Uittien & 0.07 & 0.21 & 3.83 & 5.06 & 1.50 & 1.60 & - & - \\
\hline Rhynchospora rugosa (Vahl) Galé & 4.62 & 9.75 & - & - & - & - & 3.64 & 8.46 \\
\hline Rhynchospora velutina (Kunth.) Böeck. & 0.07 & 0.43 & - & - & - & - & - & - \\
\hline Riencourtua oblongifolia Gadner & 0.10 & 0.43 & - & - & - & - & - & - \\
\hline Ruellia incompta (Nees) Lindau & - & - & 0.12 & 0.26 & - & - & - & - \\
\hline Sauvagesia linearifolia A. St.-Hil. & - & - & 0.09 & 0.52 & 2.30 & 5.66 & - & - \\
\hline Scleria hirtella Sw. & - & - & 0.10 & 0.13 & 0.14 & 0.21 & 1.08 & 3.49 \\
\hline Schultesia gracilis Mart. & - & - & - & - & 0.18 & 0.53 & - & - \\
\hline Sida cf. linifolia Cav. & - & - & 1.11 & 4.28 & 0.01 & 0.11 & - & - \\
\hline Sisyrinchium vaginatum Spreng. & 0.55 & 3.54 & 0.23 & 1.04 & 0.72 & 2.56 & - & - \\
\hline $\begin{array}{l}\text { Sporobulus reflexus } \\
\text { Boechart and Longhi-Wagner }\end{array}$ & - & - & - & - & 0.03 & 0.11 & - & - \\
\hline Stevia heptachaeta DC. & - & - & 0.30 & 1.17 & - & - & - & - \\
\hline Syngonanthus densiflorus (Körn.) Ruhland & - & - & - & - & - & - & 1.45 & 3.86 \\
\hline Syngonanthus gracilis (Bong.) Ruhland & 1.42 & 7.61 & - & - & 0.01 & 0.11 & 3.22 & 13.79 \\
\hline Syngonanthus nitens Ruhland & 0.03 & 0.43 & 0.01 & 0.13 & - & - & - & - \\
\hline $\begin{array}{l}\text { Trimezia juncifolia (Klatt.) } \\
\text { Benth. and Hook. f. }\end{array}$ & 0.33 & 3.11 & - & - & - & - & - & - \\
\hline Trimezia sp.1 & 0.03 & 0.32 & - & - & - & - & - & - \\
\hline Turnera oblongifolia Cambess. & - & - & 0.30 & 2.34 & 0.01 & 0.11 & - & - \\
\hline Wedelia bishopii H. Rob. & - & - & - & - & 0.31 & 0.85 & - & - \\
\hline Xyris guaranitica Malme & 0.39 & 1.61 & - & - & - & - & 0.03 & 0.37 \\
\hline Xyris hymenachne Mart. & - & - & - & - & 0.17 & 0.43 & - & - \\
\hline Xyris jupicai Rich. & 0.01 & 0.11 & - & - & - & - & 0.09 & 0.37 \\
\hline Xyris schizachne Mart. & 0.26 & 0.97 & - & - & - & - & - & - \\
\hline Xyris tortula Mart. & 0.04 & 0.21 & - & - & - & - & - & - \\
\hline
\end{tabular}


Zanin. in Ca1; this latter species plus Andropogon bicornis, Paspalum polyphyllum, Echinolaena inflexa (Poir.) Chase, Sauvagesia linearifolia A. St.-Hil. and Pfaffia jubata Mart. in Ca2; and Arthropogon filifolius, Rhynchospora rugosa, Arthropogon villosus Nees and Syngonanthus gracilis occurred within this range in $\mathrm{Ca} 3$. Only the latter species and Andropogon lateralis were common between two transects indicating the high variability of relative frequency at the study-site.

\subsection{Correlation vegetation $\mathrm{x}$ environmental variables}

Canonical correspondence analysis (CCA) showed strong correlations between frequency of species and environmental variables. The eigen-values were high for the two first ordination axes $(0.580$ and 0.351 respectively, explaining 13.4 and $21.5 \%$ of the species variance and 47.5 and $76.2 \%$ of the cumulative variance of the relationship species $\mathrm{x}$ environmental variables. Correlations species $\mathrm{X}$ environment at the first two axes were high (0.98 and 0.94 respectively). In addition, species frequency and environmental variables were correlated to axes 1 and 2 ( $\mathrm{F}=18.06 ; \mathrm{P}<0.01)$ according to Monte Carlo's permutation test.

The environmental variables strongly correlated to the first axis were silt, sand, clay, K, gravimetric soil moisture and $\mathrm{S}$ while the variables strongly correlated to axis 2, were $\mathrm{Fe}, \mathrm{Ca}, \mathrm{Mg}$, organic matter and $\mathrm{Al}$ (Table 3). Al presented significant weighted correlations $(>0.5)$ only with $\mathrm{pH}$ and organic matter was significantly correlated to $\mathrm{K}$ and $\mathrm{Zn}$ (Table 3). Gravimetric soil moisture presented significant weighted correlations with the majority of the soil properties except for $\mathrm{Mg}$ and organic matter. $\mathrm{P}$ was also significantly correlated to most properties except for $\mathrm{Al}$ and organic matter. The four vegetation sampling transects were clearly distinguished in both axes (Figure 1). Transect $\mathrm{Ca} 0$ occupied the lower quarter at the right hand as well as gravimentric soil moisture, $\mathrm{Ca}, \mathrm{Na}, \mathrm{Zn}$ and Fe (Figure 1). Transect $\mathrm{Ca} 3$ was separated from the others at the superior quarter at the right hand side due to high contents of organic matter and sand (Figure 1). Transects Ca1 and $\mathrm{Ca} 2$, although separated were near to the center of the left quarter of the ordination diagram; silt and clay were at an intermediate position more correlated to $\mathrm{pH}$ (Figure 1). Al occupied a central position, between the upper right quarter and the upper left, due to the high contents at the transects $\mathrm{Ca} 2$ and $\mathrm{Ca} 1$ (Figure 1).

Species ordination showed that Arthropogon villosus, Mesosetum ferrugineum (Trin.) Chase, Paspalum ellipticum Döll and Syngonanthus densiflorus (Körn.) Ruhland were strongly correlated to high organic matter (Figure 2). Ctenium brachystachyum, Drosera montana A. St.-Hil., Lagenocarpus rigidus, Rhynchospora velutina (Kunth.) Böeck. and Trimezia juncifolia (Klatt.) Benth. and Hook. f. were more correlated to $\mathrm{Ca}, \mathrm{Na}, \mathrm{Zn}$ and Fe plus gravimetric soil moisture (Figure 2). Associated to the transects on drier soils were Echinolaena inflexa, Elionurus muticus (Spreng.) Kuntze, Polygala longicaulis Humb. Bonpl. and Kunth, Pfaffia jubata and
Lessingianthus grearii (H. Rob.) H. Rob. that correlated strongly with $\mathrm{pH}$, clay and $\mathrm{Cu}$. Amongst those correlated to $\mathrm{Mn}, \mathrm{S}$ and silt, were Monnina stenophylla A. St.-Hil. and Moq., Rhynchospora graminea, Sida cf. linifolia Cav. and Turnera oblongifolia Cambess.

\section{Discussion}

Chemical analyses showed that the soils were acidic, nutrient-poor with a high aluminum saturation, therefore they were dystrophic according to the parameters considered by EMBRAPA (1999). The amplitudes of chemical variables were narrow, not sufficient to determine the edaphical variation and neither the species distribution over the study-area. Substrate conditions, especially those related to texture and other physical conditions such as soil moisture variations, organic matter and percentages of clay and sand, were the main determinants of species distribution in the moist grassland at ALF.

Transects $\mathrm{Ca} 0$ and $\mathrm{Ca} 3$ were very distinctive from $\mathrm{Ca} 1$ and $\mathrm{Ca} 2$. The first two were on sandy soils with high organic matter contents and a swampy soil over the year. $\mathrm{Ca} 1$ and $\mathrm{Ca} 2$ were on clay soils, better structured, with a seasonal humidity over the year. A larger number of species were found at $\mathrm{Ca} 1$ and $\mathrm{Ca} 2$ probably due to a betterdrained soil. Only a few species can establish and survive on anaerobic and toxic condition of swampy soils where the organic matter decomposes slowly releasing soluble Fe and Mn (Resende et al., 1997). A preferential community to permanently saturated places was characterized by some exclusive species and some preferential species. Twenty three species were restricted to the moister transects while 42 other species occurred only on the drier ones suggesting that species richness is lower in the moister soils since water saturation can limit the establishment of some species favoringonly those adapted to wetter conditions. This finding confirms Sarmiento's (1983) conclusions for Venezuelan savannas that species richness is highest on mesic conditions reducing towards extreme conditions of soil moisture either too dry or too wet. Araújo et al. (2002) also found a higher number of species at the drier than at the wetter portions of a Vereda (swampy sites dominated by Cyperaceae and grasses with lines of palms). Pinder and Rosso (1998) found species richness rising from the lower wetter sites to higher and drier sites at ten distinct phytophisiognomies at the Pantanal (wetlands) too.

A lower species richness was also found in tree communities at badly drained portions of gallery forests (Felfili, 1995, 1998). Gentry and Dodson (1987) also described higher species richness for forests on betterdrained soils. Therefore, the pattern of species richness on moist grassland follows the tendency found in several other tropical communities with a low species richness on badly drained soils.

CCA showed that the transects are heterogeneous separating clearly the transects on soils permanently saturated from those seasonally saturated. Species such as Mesosetum ferrugineum, Rhynchospora rugosa and 
Munhoz, CBR., Felfili, JM. and Rodrigues, C.

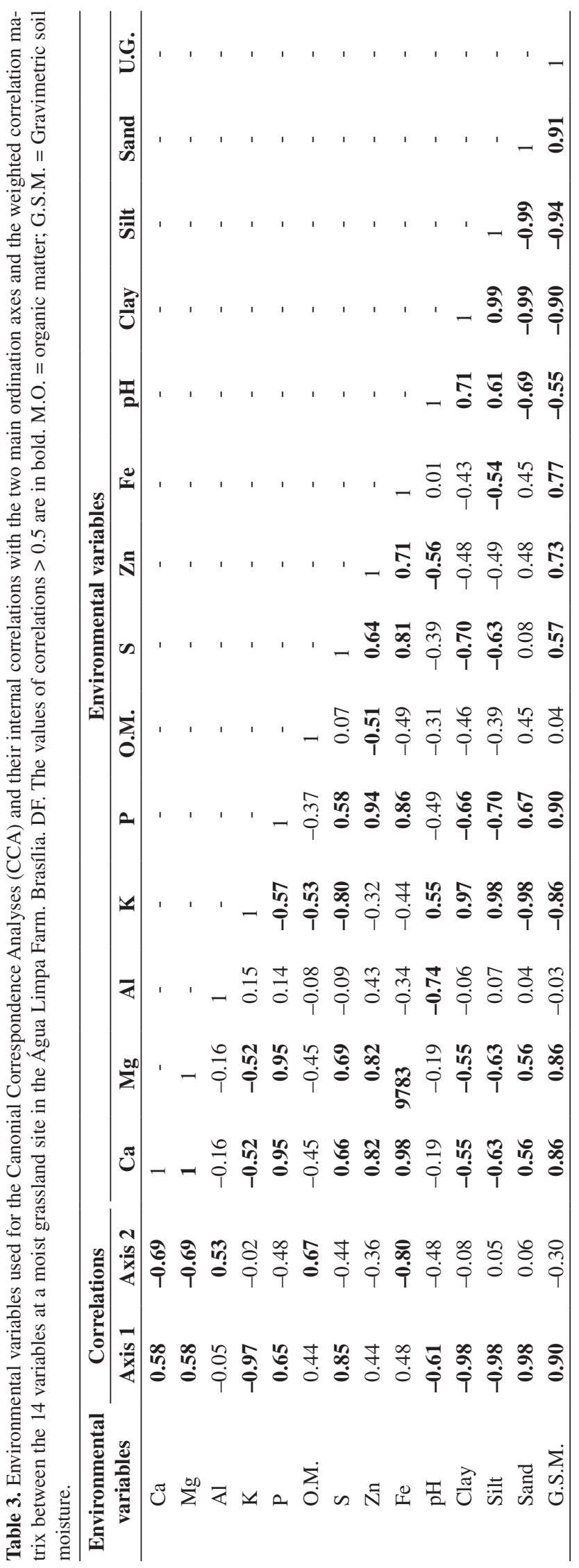




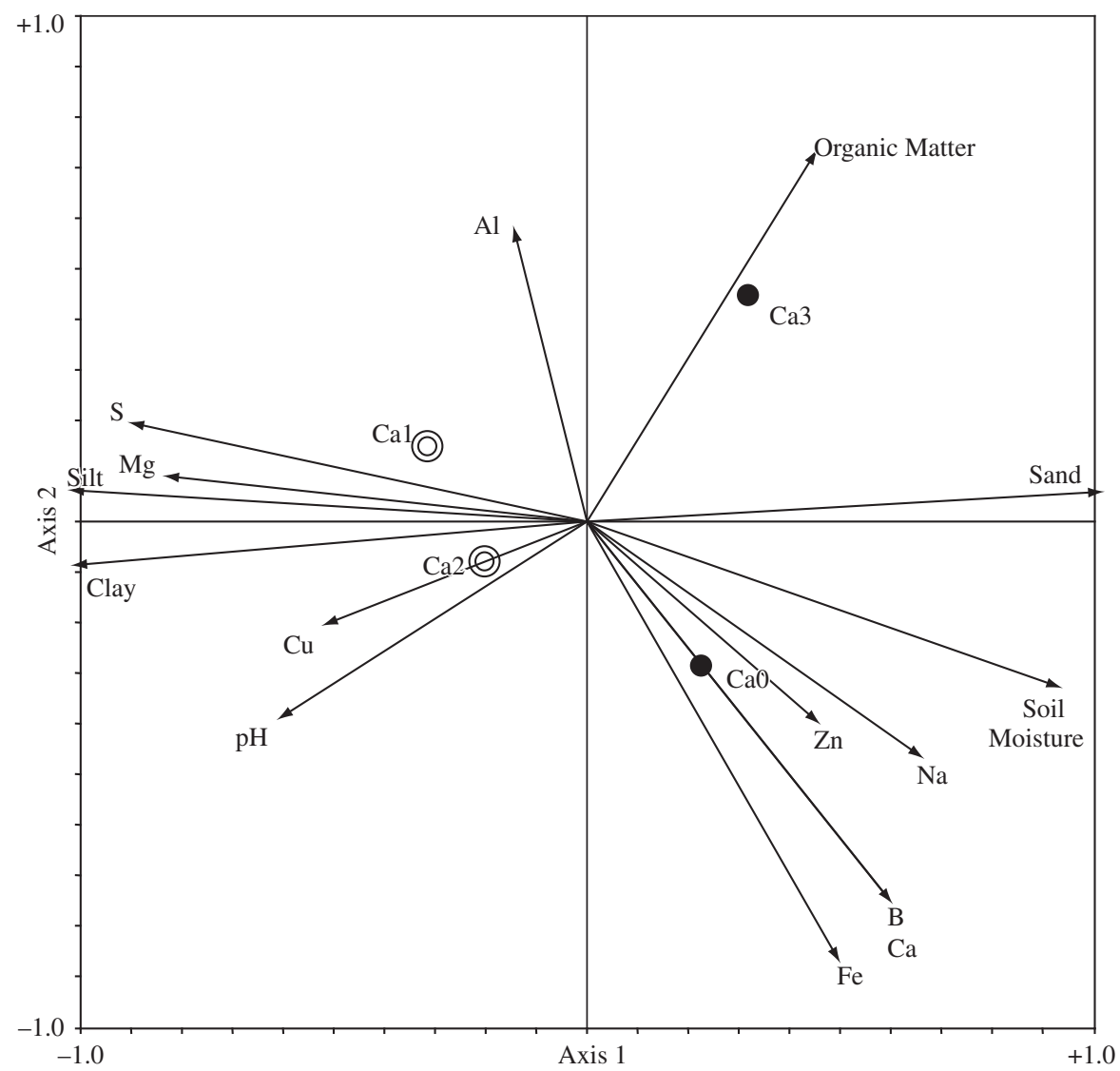

Figure 1. Ordination diagram of the sampling units and environmental variables at the first two axes produced by the Canonical corespondence analyses (CCA) for absolute frequency of the main 48 species (>10) in 120 sampling units (UA) at a moist grassland site in the Água Limpa Farm, Brasília, DF. Full circles in bold are associated to permanentely flooded transections while double empty circles are related to seasonally saturated transections.

Syngonanthus densiflorus were restricted to the transect with a swampy soil over the year being therefore good indicators of wet conditions.

The low similarity between the transects was related to the differences in the saturation of soils indicating that the moist grassland vegetation is composed of mosaics determined by soil moisture gradients even on a small spatial scale. Floyd and Anderson (1987) compared various sampling methods and suggested that the number of transects is more important for an efficient sampling of the herbaceous layer than their length. As moist grassland occurs middle way between the cerrado (savanna woodland) in the uplands and the bottom-valley, the sampling transects should cover this gradient.

Poaceae was the main family in this study and also in Goldsmith's (1974) with Andropogon, Axonopus, Mesosetum and Paspalum being abundant in both studies. Poaceae also predominated in several surveys of the herbaceous layer of cerrado sensu stricto (savanna woodland) (Mantovani and Martins, 1993; Felfili et al., 1994; Silva and Nogueira, 1999) and in Veredas (swampy grasslands with lines of palms) (Guimarães et al., 2002) espe- cially the genera Andropogon, Axonopus, Echinolaena, Paspalum and Trachypogon.

Echinolaena inflexa and Schizachyrium tenerum were the most important species of Poaceae in the cerrado sensu stricto grassy layer (Felfili et al., 1994) and in Veredas (Guimarães et al., 2002) but in this study Schizachyrium tenerum was absent while Echinolaena inflexa was poorly represented at the study-site. The main species were Axonopus comans, Andropogon lateralis subsp. cryptopus, Andropogon bicornis L. and Hypoginium virgatum (Desv.) Dandy. Axonopus comans and Andropogon lateralis subsp. cryptopus had the highest cover in the wetter parts of the moist grassland but were not found by Guimarães et al. (2002) in Veredas neither by Batalha and Martins (2002) in Campos limpos. Andropogon bicornis and Hypoginium virgatum, more common in drier portions of the moist grassland, were found in those environments by the above authors but were not found in cerrado sensu stricto (Felfili et al., 1994; Silva and Nogueira, 1999; Batalha and Martins, 2002), suggesting that those species are restricted to open-canopy and wet environment, especially the first two. 


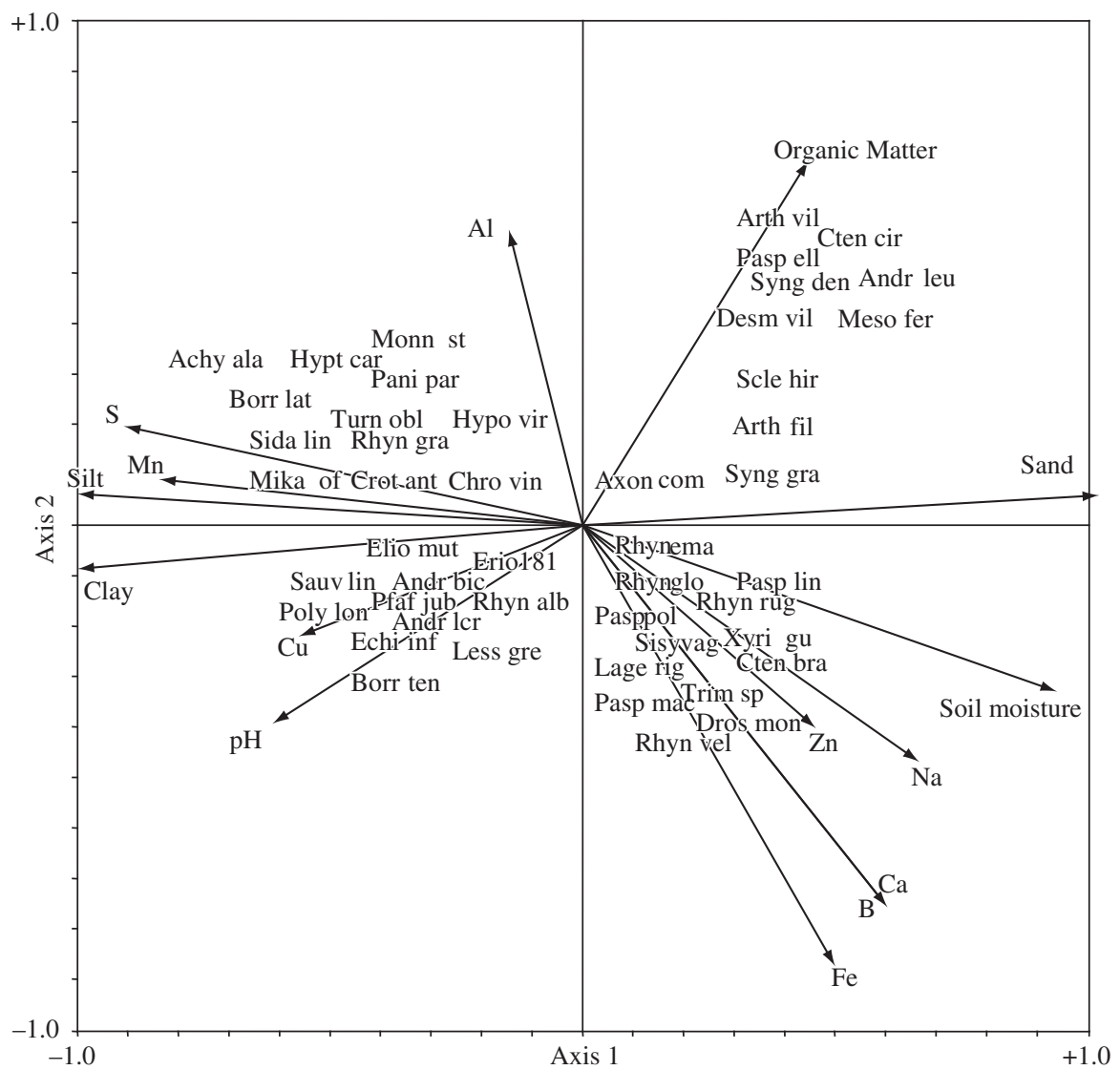

Figure 2. Ordination diagram of species and environmental variables at the first two axes produced by the Canonical corespondence analyses (CCA) for absolute frequence of the main 48 species (>10) in 120 sampling units (UA) at a moist grassland site in the Água Limpa Farm, Brasília, DF. Species are identified by the first letters of the binomial, see Table 2.

The herbaceous-shrubby layer varies widely in floristic composition from site to site (Goldsmith, 1974; Mantovani and Martins, 1993; Felfili et al., 1998) and this work shows that the variations in floristic composition are large even within a small area of moist grassland due to the soil moisture gradient. The moist grassland vegetation is composed of mosaics with a highly heterogeneous floristic composition determined by soil moisture variation related mainly to differences in soil physical variables such as texture. Soil moisture fluctuations, organic matter contents and proportions of sand and clay were the main factors determining species distribution.

The community on better drained soils was richer in species suggesting that only a small number of species can colonize permanently saturated soils. The frequent draining of moist grassland for agriculture, threatens the water sources and may lead to local extinction of species at those environments characterized by high organic matter content, high acidity and aluminium saturation and low $\mathrm{Ca}, \mathrm{P}$ and $\mathrm{Mg}$.

Acknowledgments - To Kennya Mara Oliveira Ramos and Newton Rodrigues for the support in the fieldwork. To
Christopher William Fagg for reviewing the manuscript. To CAPES for the doctorate Grant.

\section{References}

ARAÚJO, GM., BARBOSA, AAA., ARANTES, AA. and AMARAL, AF., 2002. Composição florística de veredas no município de Uberlândia, MG. Revista Brasil. Bot., vol. 25, no. 4 , p. $475-493$.

BATALHA, MA. and MARTINS, FR., 2002. The vascular flora of the cerrado in Emas National Park (Goiás, Central Brazil). Sida, vol. 20, no. 1, p. 295-312.

CANFIELD, R., 1941. Application of line interception in sampling range vegetation. J. forestry, vol. 5, no. 39, p. $388-394$.

-, 1950. Sampling range by the line interception method. Southwestern For. And Range Exp. Sta. Res. Rept., vol. 4, 28 p.

EITEN, G., 1972. The Cerrado vegetation of Brazil. Bot. Rev., vol. 38 , no. 2, p. 201-341.

EMBRAPA, 1997. Manual de métodos de análise de solo. Centro Nacional de Pesquisas de Solos: Rio de Janeiro. 
-, 1999. Sistema Brasileiro de classificação dos solos. Centro Nacional de Pesquisas de Solos, Embrapa Solos: Rio de Janeiro.

FELFILI, JM., 1995. Diversity, structure, and dynamics of a gallery Forest in Central Brazil. Vegetatio, vol. 177, no. 1, p. 1-15.

-, 1998. Determinação de padrões de distribuição de espécies em uma mata de galeria no Brasil Central com a utilização de técnicas de análise multivariada. Bol. Herb. Ezechias Paulo Heringer, vol. 2, no. 35-48.

FELFILI, JM., FILGUEIRAS, TS., HARIDASAN, M., SILVAJÚNIOR, MC., MENDONÇA, RC. and RESENDE, AV., 1994. Projeto Biogeografia do Bioma Cerrado: vegetação e solos. Cad. Geoc., vol. 12, no. 4, p. 75-166.

FELFILI, JM., SILVA-JUNIOR, MC., FILGUEIRAS, TS. and NOGUEIRA, PE., 1998. Comparison of cerrado (sensu stricto) vegetation in Brasil Central. Ciência e Cultura, vol. 50, no. 4, p. $237-243$.

FINLAYSON, CM. and VAN Der VALK, AG., 1995. Wetland classification and inventory: A summary. Vegetatio, p. 118, no. $1 / 2$, p. $185-192$.

FLOYD, DA. and ANDERSON, JE., 1987. A comparison of three methods for estimating plant cover. J. Ecol., vol. 75, no. 1, p. 221-228.

GOLDSMITH, FB., 1974. Multivariate analyses of tropical grassalnd communities in Mato Grosso, Brasil. J. Biogeography, vol. 1 , no. 1, p. 111-122.

GENTRY, AH. and DODSON, C., 1987. Contribution of nontrees to species richness of a tropical rains forest. Biotropica, vol. 19 , no. 2, p. 149-156.

GUIMARÃES, AJM., ARAÚJO, GM. and CORRÊA, GF., 2002. Estrutura fitossociológica em área natural e antropizada de uma vereda em Uberlândia, MG. Acta Bot. Bras., vol. 16, no. 3, p. 317-330.

HARIDASAN, M., 1996. Estresse nutricional, p. 27-30. In DIAS, BFS., (ed.). Alternativas de desenvolvimento dos cerrados: Manejo e conservação dos recursos naturais renováveis. FUNATURA, Brasília.

KENT, M. and COKER, P., 1992. Vegetation description and analysis; a pratical Approach. Belhaven Press, London, 363 p.

MANTOVANI, W. and MARTINS, FR., 1993. Florística do Cerrado na reserva biológica de Moji Guaçu, SP. Acta Bot. Bras., vol. 7, n. 1, p. 33-60.
MARGURRAN, AE., 1988. Ecological diversity and its measurements. Croom Helm. London, 179 p.

MENDONÇA, RC., FELFILI, JM, WALTER, BMT, SILVA JÚNIOR, MC., REZENDE, AV., FILGUEIRAS, TS. and NOGUEIRA, PE., 1998. Flora vascular do Cerrado, p. 289-556. In SANO, SM. and ALMEIDA, SP. (eds.). Cerrado: Ambiente e Flora, EMBRAPA-CPAC, Brasília.

MUELLER-DOMBOIS, D. and ELLENBERG, H., 1974. Aims and methods of vegetation ecology. Willey and Sons, New York, $547 \mathrm{p}$.

NARANJO, LG., 1995. An evaluation of the first inventory of South American wetlands. Vegetatio, vol. 118, no. 1/2, p. $125-129$.

PINDER, L. and ROSSO, S., 1998. Classification and ordination of plant formations in the Pantanal of Brazil. Plant Ecol., vol. 136, no. 2, p. 151-165.

RATTER, JA, RIBEIRO, JF and BRIDGEWATER, S., 1997. The Brasilian cerrado vegetation and threats to its biodiversity. Ann. Bot., vol. 80, no. 3, p. 223-230.

REATTO, A., CORREIA, JR. and SPERA, ST., 1998. Solos do bioma cerrado: aspectos pedológicos, p. 47-88. In SANO, SM. and ALMEIDA, SP. (eds.). Cerrado: Ambiente e Flora, EMBRAPA-CPAC, Brasília.

RESENDE, M., CURI, N., REZENDE, SB. and CORRÊA, GF., 1997. Pedologia: base para distinção de ambientes. NEPUT, Viçosa, $367 \mathrm{p}$.

RIBEIRO, JF. and WALTER, BMT., 1998. Fitofisionomias do Bioma Cerrado, p. 98-166. In SANO, SM. and ALMEIDA, SP. (eds.). Cerrado: Ambiente e Flora, EMBRAPA-CPAC, Brasília.

SARMIENTO, G., 1983. The savannas of tropical America, p. 245-288. In BOURLIÈRE, F. (ed.). Ecosystems of the world: tropical savannas, Elsevier, Amsterdam.

SILVA, MA. and NOGUEIRA, PE., 1999. Avaliação fitossociológica do estrato arbustivo-herbáceo em cerrado stricto sensu após incêndio acidental, no Distrito Federal, Brasil. Bol. Herb. Ezechias Paulo Heringer, vol. 4, p. 65-78.

SMILAUER, P., 1992. CANODRAW: User's guide., version 3.0. Microcomputer Power, Ithaca, New York, 118 p.

TER BRAAK, CJF. and SMILAUER, P., 1998. CANOCO Reference manual and user's guide to Canoco for Windows: software for canonical community ordination., version 4. Microcomputer Power, Ithaca, New York, 351 p. 
